\title{
Governança regional do sistema de saúde no Brasil: configurações de atores e papel das Comissões Intergovernamentais
}

\author{
Regional governance of the health system in Brazil: configurations \\ of actors and the role of the Interagency Commissions
}

\author{
Mariana Vercesi de Albuquerque ${ }^{1}$ \\ Luciana Dias de Lima ${ }^{1}$ \\ Ricardo Antunes Dantas de Oliveira ${ }^{2}$ \\ João Henrique Gurtler Scatena ${ }^{3}$ \\ Nereide Lucia Martinelli ${ }^{3}$ \\ Adelyne Maria Mendes Pereira ${ }^{1}$
}

${ }^{1}$ Departamento de Administração e

Planejamento em Saúde, Escola Nacional de Saúde Pública Sérgio Arouca, Fiocruz. R. Leopoldo Bulhões 1480/709,

Manguinhos. 21041-210 Rio de Janeiro RJ Brasil. mariana.albuquerque@ensp. fiocruz.br

${ }^{2}$ Instituto de Informação e Comunicação Científica e Tecnológica em Saúde, Fiocruz. Rio de Janeiro RJ Brasil.

${ }^{3}$ Instituto de Saúde Coletiva, Universidade Federal do Mato Grosso. Cuiabá MT Brasil.

\begin{abstract}
The analytical focus is on the role of the Regional Interagency Commissions (CIR), considering the diversity of actors that influence health policy in specific regional contexts. The research involved conducting five case studies in each of the Brazilian macroregions, with the application of 128 questionnaires to public managers, service providers and civil society representatives, between August 2015 and August 2016. The comparative perspective was adopted, by considering three analytical approaches: the configuration of actors (governmental and non-governmental; public and private) on regional decisions and conflicts, operation dynamics and contributions of commissions to health system policy and organization. The results showed the diversity of actors with a high degree of influence in the regions and the role of the Regional Interagency Commissions in policy coordination and conflict resolution. The commissions favor interagency negotiation and the organization of the Unified Health System vis-à-vis the Brazilian federative structure. However, they have limited scope as a space for regional health governance and are unable to incorporate the different configurations of public and private actors with power and influence over health decisions.
\end{abstract}

Key words Health policy, Governance, Regionalization, Unified Health System, Interagency relations
Resumo O foco da análise éo papel exercido pelas Comissões Intergestores Regionais, considerando a diversidade de atores que influenciam a politica de saúde em contextos regionais específicos. A pesquisa envolveu a realização de cinco estudos de caso em cada uma das macrorregiões brasileiras, com aplicação de 128 questionários a gestores, prestadores e representantes da sociedade civil, entre agosto de 2015 e agosto de 2016. Adotou-se a perspectiva comparada, considerando três eixos de análise: configurações de atores (governamentais e não governamentais; públicos e privados) nas decisões e conflitos regionais, dinâmica de funcionamento e atuação, e contribuiçães das comissões para a política e a organização do sistema de saúde. Verificou-se a diversidade de atores com alto grau de influência nas regiões e a função das Comissões Intergestores Regionais na coordenação das políticas e na resolução de conflitos. Estas favorecem a negociação intergovernamental e a organização do Sistema Único de Saúde frente à estrutura federativa brasileira. Contudo, possuem atuação limitada como espaço de governança regional da saúde, sendo incapazes de incorporar as diversas configurações de atores públicos e privados com poder e influência sobre as decisões de saúde. Palavras-chave Politica de saúde, Governança, Regionalização, Sistema Único de Saúde, Relações intergovernamentais 


\section{Introdução}

A partir de 1990, o termo governança se disseminou na América Latina e no Brasil, como retórica oficial de governos para designar um modo específico de governar, em associação com o debate internacional sobre a reforma do Estado e a 'nova Administração Pública'. Nesse sentido, encontra-se vinculado a uma visão prescritiva de 'boa governança', relacionada a propostas de organismos multilaterais, como o Banco Mundial e a Organização para a Cooperação e Desenvolvimento Econômico, para países periféricos ${ }^{2}$.

No âmbito acadêmico, por sua vez, e, particularmente, nos enfoques contemporâneos sobre gestão e políticas públicas, a governança também tem sido utilizada de forma cada vez mais frequente com conotações e significados múltiplos $^{3,4}$. Em geral, os estudos têm como referência as transformações nos processos decisórios e da ação pública frente às mudanças nas relações entre Estado e sociedade, ou entre governo, agentes privados e sociedade ${ }^{5}$. Valoriza-se o uso da governança como categoria de análise para descrever e explicar o exercício do poder em uma sociedade complexa ${ }^{6}$, incorporando a influência e as relações estabelecidas entre organizações e agentes diversos sobre a política pública, em contextos institucionais específicos ${ }^{1}$.

No caso dos estudos de políticas de saúde, a governança torna-se útil para a compreensão da intervenção regulatória do Estado nesse setor, marcada pela multiplicidade de atores em função dos seguintes processos: repartição dos esquemas de financiamento e provisão (de insumos e serviços) nos sistemas de saúde; descentralização para entes subnacionais (regionais e locais); transferência de funções de gestão e prestação para empresas privadas com ou sem fins lucrativos, e; execução de ações e serviços com relativa autonomia dos prestadores (incluindo os profissionais $)^{7,8}$

Partindo dessa concepção, este artigo tem como objetivo analisar o papel das Comissões Intergestores Regionais (CIR) na governança do sistema de saúde no Brasil. Tais Comissões foram constituídas em 2011, em substituição aos Colegiados de Gestão Regional (CIR), mantendo suas principais características de composição e modo de funcionamento ${ }^{9}$. As CIR configuram-se como instâncias de negociação, planejamento e gestão intergovernamental, formadas por representação das Secretarias de Estado de Saúde e do conjunto de municípios que compõem as regiões de saúde no âmbito do Sistema Único de Saúde (SUS).
A implantação dessas comissões foi gradual e heterogênea no território nacional, marcada pela influência de fatores socioeconômicos e da estrutura do sistema de serviços de saúde ${ }^{10}$, bem como de legados de políticas prévias e das características do processo político em âmbito estadual ${ }^{11}$. Estudo de caso no estado do Rio de Janeiro mostrou que a capacidade de atuação da CIR depende do conhecimento técnico dos gestores sobre as políticas nacionais e estaduais e seu engajamento político para a construção de regiões que efetivamente respondam às necessidades regionais ${ }^{12}$. Outra pesquisa recente, realizada na região de $\mathrm{Vi}$ tória da Conquista (BA), sugere que os conflitos presentes no processo decisório, a coordenação técnica incipiente, a rotatividade dos gestores e a carência de instrumentos regionais de planejamento e financiamento afetam o funcionamento e a atuação da $\mathrm{CIR}^{13}$. Embora estes estudos avancem na identificação dos condicionantes, das características e dos desafios do funcionamento e atuação da CIR, ainda permanecem lacunas para a compreensão do papel exercido por essas comissões, considerando a diversidade de atores que influenciam a política de saúde no espaço regional.

Assim, a pesquisa foi orientada pelas seguintes questões: Como configurações diversas de atores condicionam a dinâmica de funcionamento e atuação das CIR? Quais as contribuições dessas comissões para a política e a organização do sistema de saúde nas regiões? Presume-se que as dinâmicas de funcionamento e atuação, assim como as contribuições das CIR variem em função de diferentes composições de atores que caracterizam as relações de poder, os conflitos e o processo decisório da política de saúde nas regiões, não restritas às representações governamentais com assento nessas comissões.

\section{Métodos}

O presente artigo é um recorte de uma pesquisa multicêntrica nacional, aprovada pelo Comitê de Ética da Faculdade de Medicina da Universidade de São Paulo e cuja metodologia é detalhada por Viana et al. ${ }^{14}$. A pesquisa envolveu a realização de cinco estudos de caso, entre agosto de 2015 a agosto de 2016, englobando sete regiões de saúde selecionadas em cada uma das macrorregiões brasileiras: Baixada Cuiabana (Mato Grosso MT); Barretos Norte e Barretos Sul (São Paulo - SP); Carbonífera/Costa Doce (Rio Grande do Sul - RS); Manaus/Alto Rio Negro (Amazonas 
-AM); Juazeiro (Bahia -BA) e Petrolina (Pernambuco - PE).

Este artigo enfocou o papel das CIR na governança do sistema de saúde nas referidas regiões. Trata-se de estudo de natureza exploratória, de abordagem qualitativa, tendo como base empíri$\mathrm{ca}$ as respostas de 128 informantes-chave a questionários específicos. Tais instrumentos, semiestruturados e com foco em política, estrutura, organização e intersetorialidade, em suas distintas apresentações, foram aplicados a gestores, prestadores e representantes da sociedade civil, dos níveis municipal, regional e estadual. Para fins deste estudo, foram buscadas as questões que, no seu enunciado ou nas opções de resposta, faziam alusão à CIR.

O estudo foi desenvolvido com base em três eixos de análise. No primeiro eixo, considerando a Influência nas decisões de saúde e os Tipos de conflitos regionais, foram identificados os atores (governamentais e não governamentais, públicos e privados) que exercem alta influência nas decisões sobre saúde na região e que estão envolvidos nos conflitos regionais. Buscou-se caracterizar as configurações de atores regionais, a partir das seguintes categorias: organizações governamentais com representação nata na CIR (secretarias municipais/Conselho de Secretarias Municipais de Saúde - Cosems, e secretaria estadual/instância estadual regional); organizações e agentes do Estado sem representação nata na CIR (Ministério da Saúde, secretarias de saúde de fora da região, Tribunal de Contas, deputados/vereadores, órgãos do sistema judiciário, Ministério Público); prestadores de serviços de saúde (públicos e privados, lucrativos ou não); e organizações e agentes da sociedade (Conselhos de Saúde; mídia; usuários; organizações da sociedade civil; sindicatos e associações corporativas/profissionais; partidos políticos).

As questões que trataram dos conflitos envolveram as relações intergovernamentais (incluindo a instância de representação da secretaria estadual na região), e as relações público-privadas no âmbito de distintos elencos e cenários (atores governamentais e não governamentais, prestadores de serviços de saúde, planos de saúde suplementar, organizações profissionais e conselhos de saúde). As configurações de atores regionais foram identificadas com base na diversidade dos atores com alto grau de influência nas decisões de saúde e envolvidos nos conflitos regionais.

O segundo eixo abordou a caracterização da dinâmica de funcionamento e atuação da CIR propriamente dita. Para isso, foram consideradas as seguintes variáveis apreendidas dos questionários: o caráter da comissão (deliberativo ou consultivo); a organização interna (em comissões ou grupos de trabalho); órgãos relevantes da definição da pauta; e a interação entre os participantes (objetivos acordados ou não entre os participantes).

No terceiro eixo, procurou-se explorar as contribuições da CIR para a política e a organização do sistema de saúde em âmbito regional. As variáveis consideradas nesse eixo incluíram: a natureza dos conflitos nas negociações regionais (recursos e organização da rede assistencial); a alta relevância da atuação da CIR em relação a determinados aspectos da política e da organização do sistema de saúde no âmbito regional; e os temas referentes às redes de atenção à saúde negociados na CIR.

Ao final, a partir da análise conjunta dos três eixos considerados no estudo - configurações de atores regionais, dinâmica de funcionamento e atuação, e contribuições da CIR - e da comparação entre os casos, procurou-se discutir o papel, bem como os limites e as possibilidades da CIR na governança do sistema de saúde, considerando diferentes contextos regionais.

\section{Resultados}

\section{Configurações de atores nas decisões e conflitos regionais}

Foram identificados diversos atores com alto grau de influência nas decisões de saúde nas regiões analisadas. Em todas elas, a estrutura regional das Secretarias Estaduais de Saúde destacou-se como o principal organizador da região de saúde (Quadro 1). Das organizações com representação nata na CIR, secretarias estaduais e municipais de saúde exerceram alto grau de influência regional. Os Conselhos de Secretarias Municipais de Saúde também foram influentes em todas as regiões, com exceção da Carbonífera/Costa Doce.

Das organizações governamentais sem representação nata na CIR, o Ministério da Saúde apareceu como altamente influente em todas as regiões. Em Petrolina/Juazeiro houve influência de secretarias municipais de saúde de fora da região. Destacou-se também a influência do Tribunal de Contas em todas as regiões e de parlamentares na maioria das regiões (Baixada Cuiabana, Barretos e Petrolina/Juazeiro). Órgãos do poder judiciário foram influentes nas três regiões supramencionadas. Já o Ministério Público exerceu alto grau 


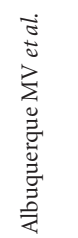

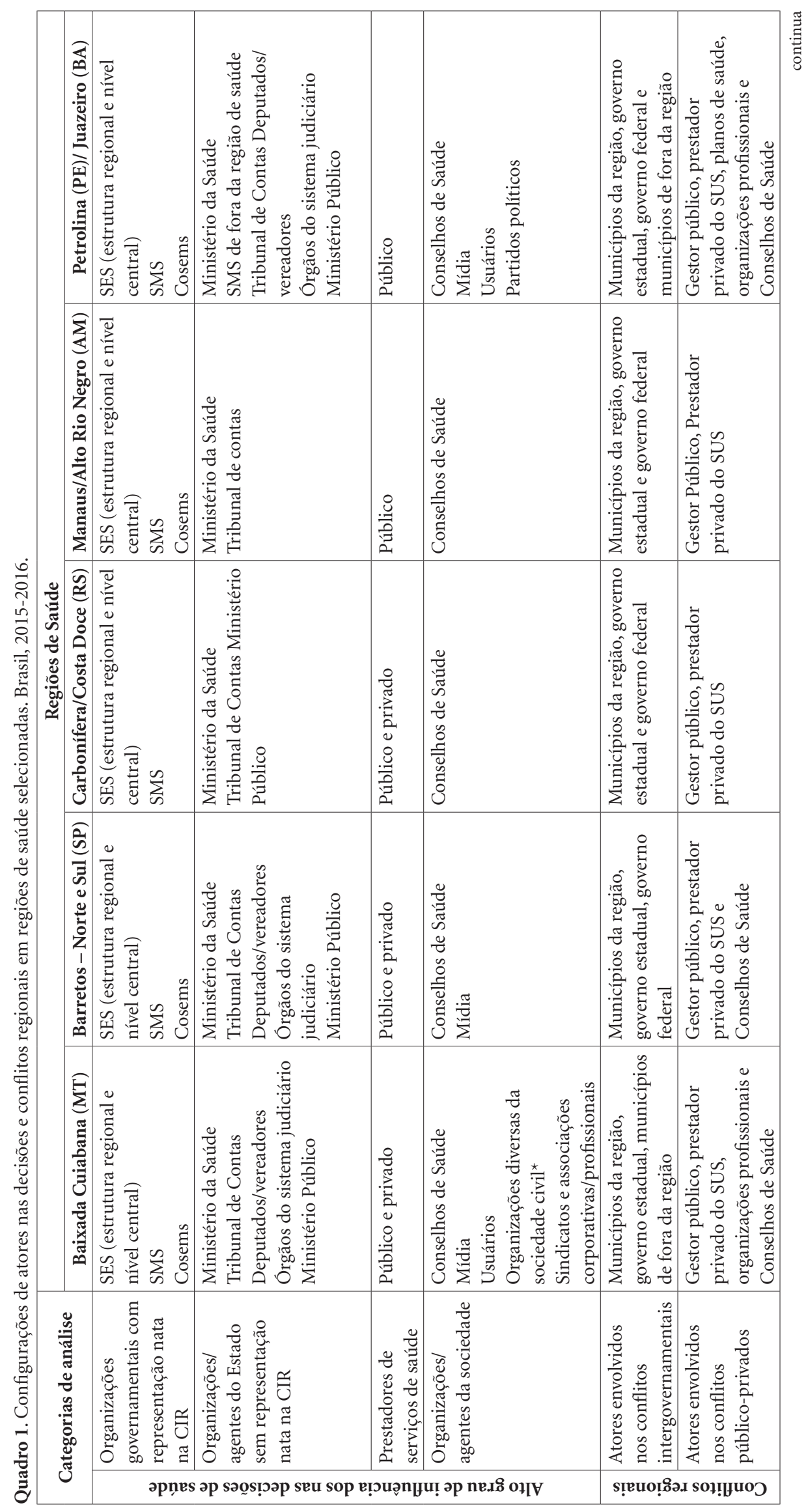




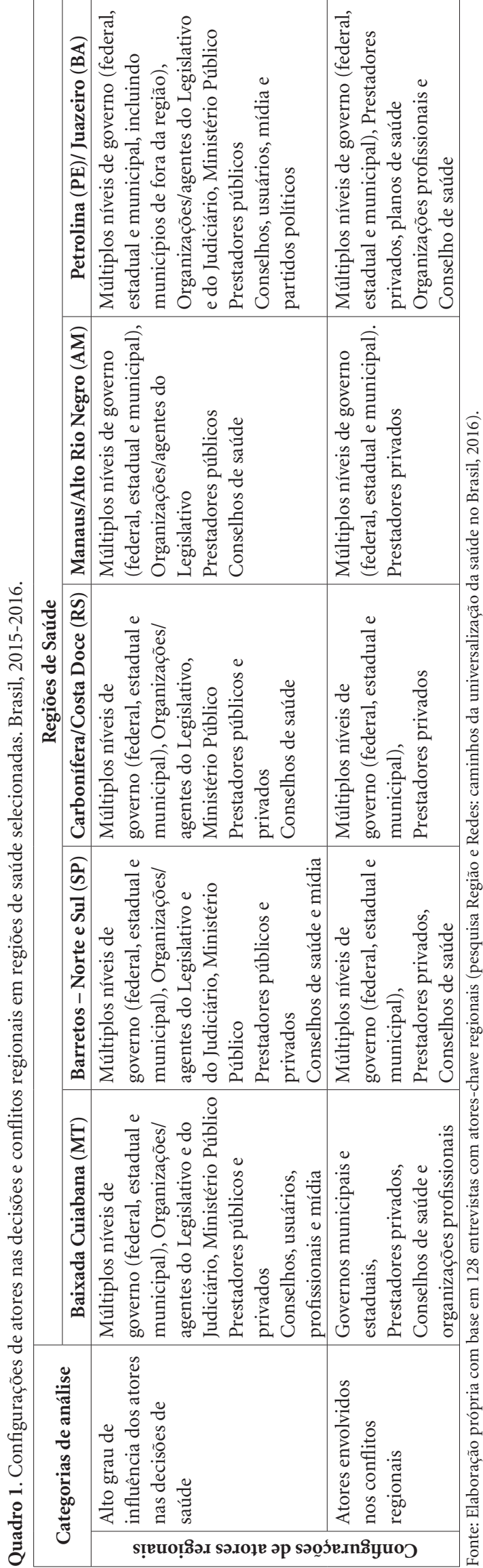

de influência em todas as regiões, exceto Manaus/ Alto Rio Negro.

Os estabelecimentos públicos, prestadores de serviços assistenciais, exerceram alto grau de influência nas decisões de saúde no conjunto de regiões de estudo, sendo que na Baixada Cuiabana, na Carbonífera e em Barretos tal influência foi partilhada com prestadores privados vinculados ao SUS.

Em relação a organizações e agentes da sociedade, a Mídia apareceu com alto grau de influência sobre as decisões de saúde em três regiões (Baixada Cuiabana, Barretos e Petrolina/ Juazeiro). Usuários e organizações da sociedade civil foram influentes em duas regiões (Baixada Cuiabana e Petrolina/Juazeiro). Os Conselhos de Saúde exerceram grande influência nas decisões de saúde em todas as regiões estudadas. Sindicatos ou associações corporativas/profissionais destacaram-se na Baixada Cuiabana e partidos políticos foram influentes em Petrolina/Juazeiro.

No que tange aos conflitos referentes às negociações e decisões de saúde nas regiões, do ponto de vista das relações intergovernamentais, para além dos municípios e dos governos estaduais, foram citados municípios de fora da região de saúde (Baixada Cuiabana Petrolina/Juazeiro) e o governo federal (todas, com exceção da Baixada Cuiabana). Já quando o foco eram as relações público-privadas, os conflitos envolveram os gestores públicos e os prestadores privados vinculados ao SUS, em todas as regiões. Em três delas foram citados também os Conselhos de Saúde (Baixada Cuiabana, Barretos e Petrolina/Juazeiro), em duas regiões destacaram-se as organizações dos profissionais de saúde (Baixada Cuiabana e Petrolina/Juazeiro) e apenas nessa última foram citados conflitos envolvendo os planos de saúde (Quadro 1).

\section{Dinâmica de funcionamento e atuação das Comissões Intergestores Regionais}

Em todas as regiões, a Comissão Intergestores Regional (CIR) e a Bipartite (CIB) foram destacadas com alto grau de influência nas decisões sobre saúde, assim como a CIT (neste caso, excetuando-se Manaus/Alto Rio Negro), e o Grupo Condutor da Rede de Atenção (ou similar) de Barretos e Petrolina/Juazeiro.

As CIR analisadas tiveram caráter deliberativo e no processo decisório predominou a realização dos objetivos acordados entre os participantes. Elas se diferenciaram quanto à organização e aos órgãos relevantes na definição das pautas. 
Na maior parte das regiões a CIR contava com comissões e/ou grupos de trabalho (Baixada Cuiabana, Carbonífera/Costa Doce e Petrolina/ Juazeiro). Quanto aos atores e instituições relevantes na definição da pauta, além da estrutura regional da SES e das secretarias municipais de saúde, destacaram-se: o apoiador do Cosems, em três regiões (Carbonífera/Costa Doce, Manaus/ Alto Rio Negro e Petrolina/Juazeiro); o apoiador do MS e as instituições de pesquisa, em Manaus/ Alto Rio Negro; e os prestadores de serviços, em Petrolina/Juazeiro.

\section{Contribuições das Comissões Intergestores Regionais para a política e a organização do sistema de saúde}

Em todas as regiões, a CIR teve grande importância para a coordenação da política de saúde, para a resolução de conflitos intergovernamentais e para o planejamento regional, monitoramento e avaliação das políticas e do sistema de saúde (Quadro 2). Com exceção da região de Manaus/ Alto Rio Negro, a CIR contribuiu em todas as regiões para a negociação do financiamento e dos aspectos para a conformação das redes de atenção à saúde. Somente em duas regiões (Carbonífera/ Costa Doce e Petrolina/Juazeiro) a CIR foi citada como um espaço importante para as negociações entre instituições públicas e privadas.

Quando o tópico são as redes de atenção à saúde (RAS), na maioria das regiões (exceção Manaus/Alto Rio Negro) a CIR contribuiu para negociações em torno dos principais temas: escopo de atuação dos prestadores, financiamento, logística e regulação do acesso. As negociações visando à articulação da rede foram citadas em três regiões (Barretos, Carbonífera e Petrolina/ Juazeiro), enquanto aquelas envolvendo as relações público-privadas que permeiam as RAS o foram em duas (Baixada Cuiabana e Petrolina/ Juazeiro), assim como as negociações relativas a recursos humanos para atenção primária em saúde (Barretos e Petrolina/Juazeiro).

Quanto aos conflitos entre os municípios da região e entre estes e as estruturas regionais das secretarias estaduais, as contribuições da CIR incidiram sobre aqueles que permeavam as decisões de saúde relacionadas principalmente a: recursos, critérios de acesso e organização das redes.

\section{Discussão}

A participação crescente de atores não governamentais, sobretudo de capital privado, nas políticas e nos sistemas de saúde é um fenômeno mundial e tornou mais complexa a governança da saúde ${ }^{7,8,15}$. No Brasil, este fenômeno veio acompanhado pela diversificação das formas de inserção dos atores não governamentais ${ }^{16} \mathrm{e}$ pela dominância financeira no setor ${ }^{17}$. E cada região de saúde sustenta e explica um conjunto de ações localizadas e interdependentes, com interesses e formas de atuação diversas, que, pela coexistência, participam e/ou influenciam o exercício do poder no território ${ }^{18}$.

Nas regiões estudadas foram encontradas diversas configurações de atores - governamentais e não governamentais, direta ou indiretamente relacionados com o setor saúde - com alto grau de influência na política e no sistema de saúde, o que enfatiza a importância de estudos sobre a governança na perspectiva regional.

A configuração dos atores varia nas regiões de saúde segundo as organizações e as instituições sem representação nata na CIR. Configurações mais diversas incluem os poderes legislativo e judiciário, o Ministério Público, o Tribunal de Contas e os municípios de fora da região, além de prestadores privados, da mídia, de usuários, de profissionais e de partidos políticos. Na comparação das cinco regiões foram encontradas configurações diversas, o que sugere o potencial da CIR para expressar distintas realidades regionais. Baixada Cuiabana e Petrolina/Juazeiro representam a maior diversidade de atores com influência regional e, no extremo oposto, está a região de Manaus/Alto Rio Negro.

Do ponto de vista de organizações e agentes da sociedade, chama a atenção o fato de os Conselhos de Saúde terem sido citados como altamente influentes nas cinco regiões. Por um lado, esse resultado demonstra a importância da existência desses espaços de participação social constituídos no âmbito da política de saúde no país. Por outro, sabe-se que existem limitações quanto à efetiva influência exercida pela população através dos Conselhos de Saúde, por conta de estratégias neocorporativas e cooptação de seus representantes por parte do Estado ${ }^{19}$. Diante da atual complexidade dos arranjos de governança e da diversidade de atores com influência no setor talvez seja possível pensar novas estratégias para a ampliação da participação da população nas negociações em escala regional. 


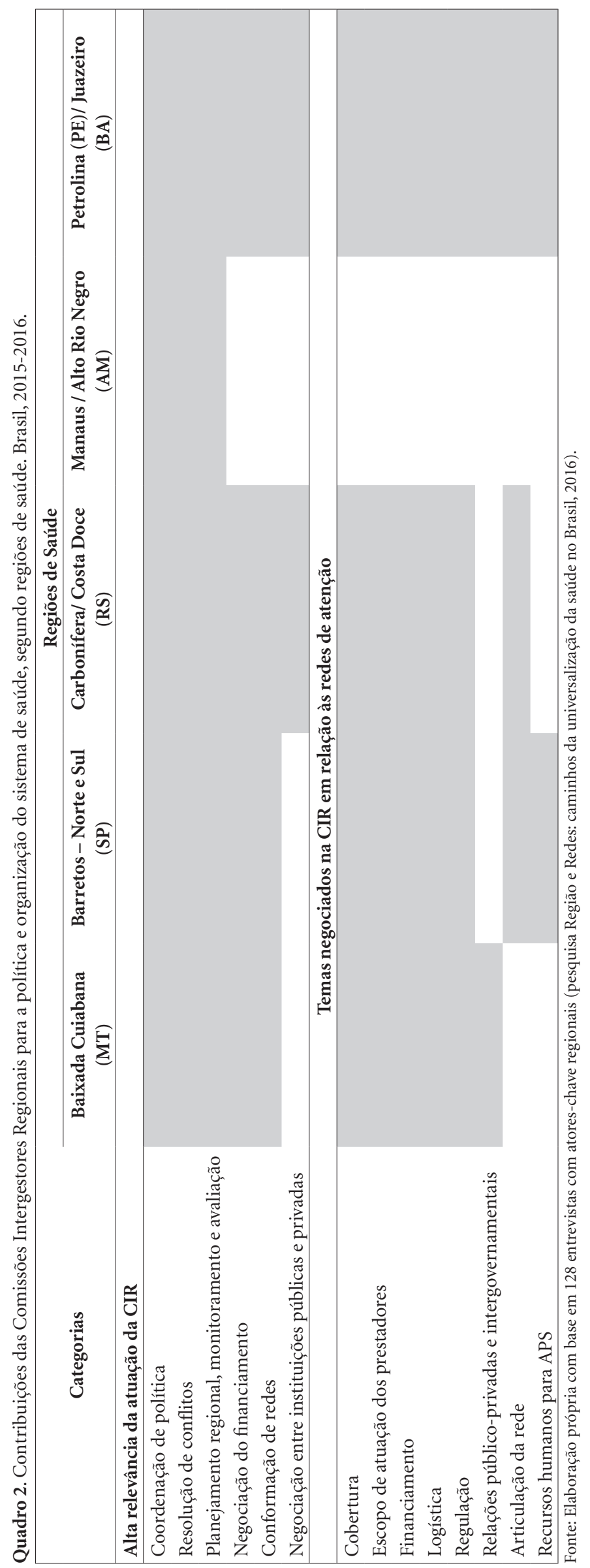


Do ponto de vista de organizações e agentes do Estado sem representação nata na CIR, destaca-se a alta influência do Ministério da Saúde e do Tribunal de Contas dos estados nas decisões de saúde em todas as regiões analisadas. Esse resultado reafirma a importância da indução federal na política de saúde e organização do sistema, conforme apontado por outros estudos ${ }^{20-22}$. Ressalta-se o papel exercido pelos Tribunais de Contas na regulação da política de saúde nas escalas municipal e regional, e que foi pouco estudado nesta perspectiva.

A influência exercida pelos prestadores públicos e privados na maior parte das regiões condiz com a tendência de expansão do setor privado credenciado ao SUS em regiões com maior dinamismo econômico ${ }^{23}$ e da crescente dependência e subordinação da política de saúde aos interesses privados no setor na escala regional ${ }^{13,24,25}$. O processo de regionalização do SUS não impediu a crescente privatização do setor e não representou maiores avanços para a atuação do Estado, sobretudo no planejamento e regulação $0^{12,13,25,26}$. Tais tendências apontam para configurações cada vez mais diversas dos atores, que tornam mais complexo e conflituoso o processo de negociação e decisão em torno da política de saúde nas regiões.

Viana et al. ${ }^{27}$ definiram três tipos de arranjos de governança para as mesmas cinco regiões de saúde deste estudo, considerando a interdependência de funções e recursos entre esferas de governo e prestadores públicos e privados na assistência especializada do SUS. Na comparação com os resultados aqui apresentados, verificou-se que as regiões com as configurações mais diversas dos atores influentes são aquelas cujos tipos de arranjos de governança envolvem grande interdependência entre as esferas municipal e estadual, do polo regional e dos prestadores públicos e privados nas decisões de saúde na região. Já a região com a configuração menos diversa (Manaus/ Alto Rio Negro) é aquela cujo tipo de arranjo de governança se caracteriza pela interdependência entre esfera estadual, polo regional e prestadores públicos nas decisões de saúde, isto é, um rol menos diverso de atores condiz com um arranjo de governança mais restrito ao papel do Estado e dos prestadores públicos. Compreende-se, assim, que quanto mais diversificado e complexo o quadro dos atores com influência nas decisões de saúde na região, maior tende a ser a interdependência entre diferentes esferas de governo, prestadores públicos e privados. Esses arranjos podem se expressar, com maior ou menor intensidade, na CIR.
A diversidade das regiões de saúde é tanto condicionada pelas suas características socioeconômicas e de oferta de serviços de saúde ${ }^{10}$, quanto pela configuração dos atores com influência no setor. A diversidade reside na combinação de atores, ações e situações (de vida e de oferta assistencial) em cada região de saúde.

A multiplicidade de atores governamentais e não governamentais, públicos e privados que exercem alto grau de influência também se reflete nos conflitos relativos às decisões de saúde em cada região. Considerando a lógica de distribuição territorial de oferta assistencial de alta e média complexidade, extremamente concentrada em poucos polos regionais ${ }^{27}$, a natureza dos conflitos é a mesma em todas as regiões: recursos, critérios de acesso e organização das redes. Nem todos os atores envolvidos em conflitos e disputas nas regiões de saúde têm representação nata na CIR, embora participem eventualmente das reuniões. Apesar de em todas as regiões a atuação da CIR ser considerada como de alta relevância para a resolução de conflitos regionais, apenas em duas (Carbonífera/Costa Doce e Petrolina/ Juazeiro) foi mencionada também sua alta relevância para a negociação entre instituições públicas e privadas. No âmbito das CIR, a questão é discutida nas regiões da Baixada Cuiabana, Carbonífera/Costa Doce e Petrolina/Juazeiro, porém fica claro que a negociação a respeito das decisões de saúde nas regiões passa por outros canais e atores além daqueles formalmente representados na CIR, ainda que não seja possível mensurar o quanto os secretários municipais e estaduais estão levando para as CIR os interesses e as demandas desses outros atores. Certamente, as CIR podem ser mais ou menos abertas à influência de atores externos, como no caso da região de Petrolina/Juazeiro, onde prestadores foram citados como influentes na pauta da CIR.

Em geral, o funcionamento e a organização das CIR são semelhantes em todas as regiões: caráter deliberativo; interação entre os participantes marcada pela realização dos objetivos acordados; e papel dos membros natos na definição da pauta. Distinguem-se pelos agentes e instituições externos à CIR que contribuem à pauta e pela forma de organização do trabalho.

Quando consideradas as contribuições da CIR, observa-se que na maioria das regiões sua atuação tem alta relevância para a negociação de temas diversos, para o planejamento e a coordenação/cooperação intergovernamental, incluindo os temas negociados em relação às redes de atenção. Distingue-se a região de Manaus/Alto 
Rio Negro, onde a atuação da CIR envolve menor diversidade de temáticas e há pouca negociação sobre as redes de atenção. Problemas estruturais de logística e insuficiência da rede assistencial enfrentados na Amazônia Legal dificultam tanto a conformação e a atuação das CIR quanto a implementação das redes de atenção ${ }^{26,28}$.

De modo geral, as redes de atenção demandam muitas discussões e negociações a respeito de seu complexo modelo de implantação e operacionalização, valorizando a atuação da CIR. Contudo, esta instância precisa incorporar outros atores para dar conta da governança das redes de atenção ${ }^{26,29,30}$. Sobretudo observando que a atuação da CIR tende a ser considerada mais relevante para aspectos da política e organização do sistema de saúde em regiões marcadas pela maior diversidade de atores que exercem influência no setor. As regiões de Petrolina/Juazeiro e Baixada Cuiabana ilustram essa percepção, enquanto a região Carbonífera/Costa Doce é um exemplo contrário. Neste caso, a insuficiência de serviços na própria região e a dependência de outra é uma possível razão para o destacado papel da CIR em muitas temáticas, mesmo com a menor diversidade e complexidade dos atores.

Quando se compreende a federação numa perspectiva mais ampla do exercício do poder para além do Estado $^{18}$ e que a negociação em torno da política de saúde envolve uma complexa configuração de atores, ações e interesses públicos e privados, percebe-se que a CIR possui atuação limitada como espaço de governança regional da saúde. Tal fato ajuda a explicar as dificuldades que estas comissões possuem em romper com lógicas estabelecidas de disputa por recursos escassos entre entes governamentais e de subordinação aos interesses privados ${ }^{13,24-26,31}$. Embora favoreça a negociação intergovernamental, a CIR não é capaz de incorporar as diversas configurações de atores públicos e privados que fazem parte do sistema de saúde e que exercem poder e influências nas regiões. Além de outros atores indiretamente relacionados ao setor saúde e que são centrais no processo de negociação regional ${ }^{25}$. A CIR também não estabelece novos mecanismos, estratégias e projetos para dar conta da complexa governança da saúde em diferentes contextos regionais.
A diversidade e a influência dos atores no sistema de saúde no âmbito regional podem limitar a atuação da CIR. Muitas vezes as participações são pontuais, não estruturadas e produzem arranjos entre os gestores da rede de atenção municipal e regional influenciando negativamente o desenvolvimento da política de saúde regional. Tais atores, por agirem em prol de interesses particulares, não contribuem para a formação de uma identidade regional, fragmentam as ações e enfraquecem a perspectiva da cooperação e coordenação intergovernamental da regionalização ${ }^{24}$. Dificultam assim o estabelecimento de novos mecanismos, estratégias e projetos que sejam capazes de lidar com a complexa governança da saúde em diferentes contextos regionais.

A CIR pode se constituir como um importante instrumento de governança regional, como sugerem alguns estudos ${ }^{12,13,24,26}$, pois é espaço permanente de negociação intergovernamental, apesar dos fatores aqui identificados como restritivos para sua atuação. Ela cria possibilidades de compartilhamento de problemas e necessidades e de planejamento e coordenação regional do SUS, visando à cooperação intergovernamental. Contudo, é preciso lembrar que a cooperação e a coordenação regional do SUS não ocorrem apenas no plano intergovernamental. São processos que se concretizam e se fundamentam pela atuação de diferentes atores, incluindo os profissionais, os usuários, os prestadores, os consórcios, entre outros, que não estão representados na CIR e na negociação na escala regional ${ }^{24,25}$. Além disso, a proposta de conformação de redes de atenção à saúde provoca mudanças na forma de operar de cada ator regional e impõem novos desafios para a governança do SUS ${ }^{16,26,29,32}$.

É importante reconhecer as limitações da CIR frente aos novos arranjos de governança do SUS, seja para repensar e propor novos espaços e instrumentos, seja para aperfeiçoar a própria CIR, com vistas ao fortalecimento da gestão pública e à ampliação da base de sustentação do SUS $^{25-27}$. Da mesma forma, é preciso reconhecer que o papel da CIR em cada região será sempre diferente e que sua atuação estará permeada pelos interesses privados, pois expressa a própria estruturação do SUS. Conclui-se que a efetivação da CIR como um espaço de governança regional do SUS depende de sua capacidade em lidar com a diversidade de atores com influência regional. 


\section{Colaboradores}

MV Albuquerque, LD Lima e RAD Oliveira foram responsáveis pela concepção, análise das informações e redação do artigo. JHG Scatena e NL Martinelli redigiram a seção de Métodos e contribuíram com a discussão. AMM Pereira colaborou com a introdução e com a discussão. Todos foram responsáveis pela revisão final do artigo.

\section{Agradecimentos}

A pesquisa contou com recursos do Ministério da Ciência, Tecnologia e Inovação e do Ministério da Saúde, por meio da Chamada MCTI/CNPq/ CT - Saúde/MS/SCTIE/Decit. LD Lima é bolsista produtividade do Conselho Nacional de Desenvolvimento Científico e Tecnológico (CNPq).

\section{Referências}

1. Marques EC. Government, political, actors and governance in urban policies in Brazil and São Paulo: concepts for a future research agenda. Braz Political Sci Rev 2013; 7(3):8-35.

2. Sousa Santos B. A crítica da governação neoliberal: o Fórum Social Mundial como política e legalidade cosmopolita subalterna. Revista Crítica de Ciências Sociais 2005; 72:7-44.

3. Rhodes RAW. The new governance: governing without government. Political Studies 1996; XLIV:652-667.

4. Stoker G. Governance as theory: five propositions. Int Soc Sci J 1998; 50(155):17-28.

5. Menicucci TMG, Gontijo JGL, organizadores. Gestão e políticas públicas no cenário contemporâneo: tendências nacionais e internacionais. Rio de Janeiro: Editora Fiocruz; 2016.

6. Defarges PM. La Gouvernance. Paris: PUF; 2008.

7. Jakubowski E, Saltman RB, editor. The changing national role in health system governance. A case-based study of 11 European countries and Australia. Brussels: The European Observatory on Health Systems and Policies, WHO; 2013.

8. Greer SL, Jarman H, Azorsky A. A reorganisation you can see from space: the architecture of power in the new NHS. London: Centre for Health and Public Organization; 2014.

9. Brasil. Decreto ${ }^{\circ} 7.508$, de 28 de junho de 2011. Regulamenta a Lei $n^{\circ} 8.080$, de 19 de setembro de 1990, para dispor sobre a organização do Sistema Único de Saúde - SUS, o planejamento da saúde, a assistência à saúde e a articulação interfederativa, e dá outras providências. Diário Oficial da União 2011; 29 jun.

10. Viana ALA, Lima LD, Ferreira MP. Condicionantes estruturais da regionalização na saúde: tipologia dos Colegiados de Gestão Regional. Cien Saude Colet 2010; 15(5):2317-2326.

11. Machado CV, Lima LD, Viana ALA, Oliveira RG, Iozzi FL, Albuquerque MV, Scatena JHG, Mello GA, Pereira AMM, Coelho APS. Federalismo e política de saúde: comissões intergovernamentais no Brasil. Rev Saude Publica 2014; 48(4):642-650.

12. Vianna RP, Lima LD. Colegiados de Gestão Regional no estado do Rio de Janeiro: atores, estratégias e negociação intergovernamental. Physis 2013; 23(4):1025-1049.

13. Santos AM, Giovanella L. Governança regional: estratégias e disputas para gestão em saúde. Rev Saude Publica 2014; 48(4):622-631. 
14. Viana ALA, Bousquat A, Ferreira MP, Cutrim MAB, Uchimura LYT, Fusaro ER, Souza MR, Mota PHS, Pereira APCM, Iozzi FL, Albuquerque MV. Região e Redes: abordagem multidimensional e multinível para análise do processo de regionalização da saúde no Brasil. Rev. Bras. Saude Mater. Infant. 2017; 17(Supl. 1):S7-S16

15. Cataia M. Território usado e federação: articulações possíveis. Educ. Soc. 2013; 34(125):1135-1151.

16. Duran PRF, Gerschman S. Desafios da participação social nos conselhos de saúde. Saude Soc 2014; 23(3):884896.

17. Viana ALD, Machado CV. Descentralização e coordenação federativa: a experiência brasileira na saúde. Cien Saude Colet 2009; 14(3):807-817.

18. Silva RM, Andrade LOM. Coordenação dos cuidados em saúde no Brasil: o desafio federal de fortalecer a atenção primária à saúde. Physis 2014; 24(4):12071228.

19. O’Dwyer G, Konder MT, Reciputti LP, Lopes MGM, Agostinho DF, Alves GF. O processo de implantação das unidades de pronto atendimento no Brasil. Rev Saude Publica 2017; 51(11):1-12.

20. Albuquerque MV, Viana ALD, Lima LD, Ferreira MP, Fusaro ER, Iozzi FL. Desigualdades regionais na saúde: mudanças observadas no Brasil de 2000 a 2016. Cien Saude Colet 2017; 22(4):1055-1064.

21. Lima LD, Scatena JHG, Albuquerque MV, Oliveira RAD, Martinelli NL, Pereira AMM. Arranjos de governança da assistência especializada nas regiões de saúde do Brasil. Rev. Bras. Saude Mater. Infant. 2017; 17(Supl. 1):S107-S119.

22. Mendes EV. Comentários sobre as Redes de Atenção à Saúde no SUS. Divulgação em Saúde para Debate 2014; (52):38-49.

23. Oliveira CF. A Estratégia de Grupos Regionais do estado do Rio de Janeiro e seu papel no processo no processo de regionalização da saúde [dissertação]. Rio de Janeiro: Escola Nacional de Saúde Pública Sergio Arouca; 2017.

24. Casanova AO, Cruz MM, Giovanella L, Alves GR, Cardoso GCP. A implementação de redes de atenção e os desafios da governança regional em saúde na Amazônia Legal: uma análise do Projeto QualiSUS-Rede. Cien Saude Colet 2017; 22(4):1209-1224.

25. Martinelli NL, Viana ALA, Scatena JHG. O Pacto pela Saúde e o processo de regionalização no estado de Mato Grosso. Saúde em debate 2015; 39(n. spe.):76-90.
26. Goya N, Andrade LOM, Pontes RJS, Tajra FS. Regionalização da saúde: (in)visibilidade e (i)materialidade da universalidade e integralidade em saúde no trânsito de institucionalidades. Saude Soc 2016; 25(4):902-919.

27. Viana ALA, Miranda AS, Silva HP. Segmentos institucionais de gestão em saúde: descrição, tendências e cenários prospectivos. In: Noronha JC, Lima LD, Chorny AH, Dal Poz MR, Gadelha P, organizadores. Brasil Saúde Amanhã: dimensões para o planejamento da atenção à saúde. Rio de Janeiro: Fundação Oswaldo Cruz; 2017. p. 151-188.

28. Shimizu HE. Percepção dos gestores do Sistema Único de Saúde acerca dos desafios da formação das Redes de atenção à saúde no Brasil. Physis 2013; 23(4):11011122.

29. Pereira AMM, Lima LD, Machado CV, Freire JM. Descentralização e regionalização em saúde na Espanha: trajetórias, características e condicionantes. Saúde em Debate 2015; 39(n. spe.):11-27.

30. Mendes A, Louvison M. O debate da regionalização em tempos de turbulência no SUS. Saude Soc 2015; 24(2):393-402.

31. Vargas I, Mogollón-Pérez AS, Unger JP, Silva MR, De Paepe P, Vázquez ML. Regional-based integrated healthcare network policy in Brazil: from formulation to practice. Health Policy Plan 2014; 30(6):705-717.

32. Sousa ABL, Garnelo L, Mota PHS, Bousquat A. Rede regional de saúde no contexto Amazônico: o caso de Manaus, Entorno e Alto Rio Negro. Rev Bras Saúde Mater Infant 2017; 17(Supl. 1):S239-S248.

Artigo apresentado em 31/01/2018

Aprovado em 06/03/2018

Versão final apresentada em 14/05/2018 
